\title{
Simulating the effect of surfactant structure on bending moduli of monolayers
}

\author{
Live Rekviga) and Bjørn Hafskjold \\ Department of Chemistry, Norwegian University of Science and Technology, N-7491 Trondheim, Norway \\ Berend Smit \\ Department of Chemical Engineering, University of Amsterdam, Nieuwe Achtergracht 166, \\ 1018 WV Amsterdam, The Netherlands
}

(Received 30 October 2003; accepted 10 December 2003)

\begin{abstract}
We have used dissipative particle dynamics to simulate amphiphilic monolayers on the interface between oil and water. An ultralow interfacial tension is imposed by means of Monte Carlo to resemble the amphiphilic films that separate oil and water regions in microemulsions. We calculate the bending modulus by analyzing the undulation spectrum. By varying the surfactant chain length and topology we investigate the effect of surfactant structure and composition of the monolayer on the bending moduli. We find that increasing the thickness has a larger effect than increasing the density of the layer. This follows from the observations that at a given interfacial tension, the bending modulus increases with chain length and is larger for linear than branched surfactants. The increase with chain length is approximately linear, which is slower than the theoretical predictions at a fixed area. We also investigated a binary mixture of short and long surfactants compared to pure layers of the same average chain length. We find a roughly linear decrease in bending modulus with mole fraction of short surfactants. Furthermore, the mixed film has a lower bending modulus than the corresponding pure film for all mole fractions. Linking the bending moduli to the structure of the surfactants is an important step in predicting the stability of microemulsions. (C) 2004 American Institute of Physics. [DOI: 10.1063/1.1645509]
\end{abstract}

\section{INTRODUCTION}

The interaction between two interfaces of amphiphilic molecules is of importance in many systems: Membranemembrane interaction in biological systems is one example, ${ }^{1}$ another is microemulsions. ${ }^{2,3}$ Microemulsions are surfactantrich emulsions where hydrophilic and hydrophobic regions are so well mixed that the interfaces dominate. They can take on many structures such as water droplets in oil, oil droplets in water, spongelike, bicontinuous structures, and lamellar phases. To better understand the behavior of such systems we would like to relate the mesoscopic properties of the interface to the structure of the surface active molecules which constitute the interface. The relation goes via a description of the interfacial properties: the interfacial tension and the elastic constants. These properties describe the energy of an interface with a given area and principal curvatures $c_{1}$ and $c_{2},{ }^{4}$

$$
E=\int d A\left(\gamma+\frac{\kappa}{2}\left(c_{1}+c_{2}-2 c_{0}\right)^{2}+\bar{\kappa} c_{1} c_{2}\right) .
$$

Here, $\gamma$ is the interfacial tension, $\kappa$ is the bending modulus, $\bar{\kappa}$ is the saddle-splay (Gaussian) modulus, and $c_{0}$ is the spontaneous curvature. Previously we investigated the dependence of the interfacial tension $\gamma$ on surfactant structure. ${ }^{5}$ Here we examine how this structure influences the bending modulus $\kappa$.

\footnotetext{
a) Author to whom correspondence should be addressed. Electronic mail: live.rekvig@phys.chem.ntnu.no
}

The energy of an interface is, to a first approximation, characterized by its interfacial tension, which is a measure of the energy cost of increasing the interfacial area by one unit. For interfaces between oil and water, or air and water, the tension will normally be high and the two phases will be well separated. Furthermore, the interfaces are essentially flat on the scale involving hundreds of molecules. The interfacial tension can however be substantially lowered by adding a surfactant. The surfactant molecules will adsorb on the interface with their hydrophilic part in the water phase and the hydrophobic part in the oil phase. When the surfactant coverage is high, the energy cost of increasing the area of an interface can become very low. Under these circumstances the bending modulus becomes important. $\kappa$ characterizes the resistance of the interface towards bending. A low bending modulus means large thermal undulations. Such fluctuations give rise to entropic repulsive forces between two interfaces close to each other such as two bilayers in lamellar phases or two monolayers separating the oil and water regions in a microemulsion. ${ }^{6}$ Microemulsions are characterized by low interfacial tension. Their phase (oil-in-water, water-in-oil, lamellar, or bicontinuous) is therefore largely dictated by the spontaneous curvature and bending modulus. ${ }^{7}$

Experimentally, $\kappa$ can be determined using highresolution scattering techniques. ${ }^{8}$ The spectrum of undulation modes is measured and fitted to the spectrum predicted by the Hamiltonian model Eq. (1). It is now possible to compute $\kappa$ in molecular dynamics simulations in much the same way. This was first done by Goetz et al. ${ }^{9}$ who studied a lipid bi- 
layer. They found two regimes: The spectral intensity of wave lengths on the molecular scale (protrusion modes) scaled as $q^{2}$, where $q=2 \pi / \lambda$ and $\lambda$ is the undulation wavelength. The intensity of the longest wave lengths scaled as $q^{4}$, and from the intensity of these modes, $\kappa$ was extracted.

Whereas Goetz et al. used an iterative procedure to find the area corresponding to a tensionless membrane, Lindahl and Edholm ${ }^{10}$ employed a pressure scaling scheme to simulate a tensionless bilayer. This study showed an anticorrelation between area fluctuations and the intensity of undulatory modes. It therefore seems natural to use a simulation scheme that includes the natural area fluctuations when sampling the undulation spectrum. Several computational studies of bilayer rigidity have followed. ${ }^{11-13}$ Laradji and Mouritsen ${ }^{14}$ studied a monolayer on the oil/water interface with molecular dynamics. Using symmetric surfactants and special boundary conditions, they investigated the effect of surfactant density on $\gamma$ and $\kappa$. They found that $\kappa$ decreases with increasing surfactant density for low densities but increases with further increase of the surfactant density.

A few theoretical studies based on the mean-field approach have addressed the effect of structure and composition on the elastic constants in detail. ${ }^{15-19}$ The results differ qualitatively with respect to the dependence of $\kappa$ on chain length and on mole fractions of cosurfactants. The theoretical approaches differ in a few respects; one of the differences being fixed area versus fixed interfacial tension. In this paper we describe large scale particle simulations that further investigate these issues.

Previous simulation studies used molecular dynamics $9,10,14$ with a united atoms model ${ }^{10}$ or a LennardJones based model. ${ }^{9,14}$ In this simulation study we use a more coarse-grained approach: A simple model of head, tail, water, and oil beads captures the essential properties of ternary systems such as phase separation and adsorption. Changes in surfactant structure such as chain length and branching can easily be realized. We calculate bending moduli for a variety of surfactant structures and binary mixtures of surfactants. The aim is a molecular understanding of the bending modulus. This is important because it is agreed that the bending modulus is a key parameter in understanding structure and phase behavior of microemulsions. ${ }^{20}$

We have chosen a coarse-grained method, dissipative particle dynamics (DPD) to govern the particle dynamics. The advantage of DPD over molecular dynamics is that the accessible time and length scales are increased by approximately an order of magnitude. We use a Monte Carlo technique to vary the box shape during the simulations according to a specified constant interfacial tension. ${ }^{21}$ In this manner we can compare the bending moduli for monolayers at the same interfacial tension, resembling saturated, low-tension layers such as in a microemulsion.

\section{MODEL AND SIMULATION DETAILS}

\section{A. Dissipative particle dynamics}

In DPD, conservative, random, and dissipative forces act between two particles $i$ and $j$ which are a distance $r_{i j}$ apart,

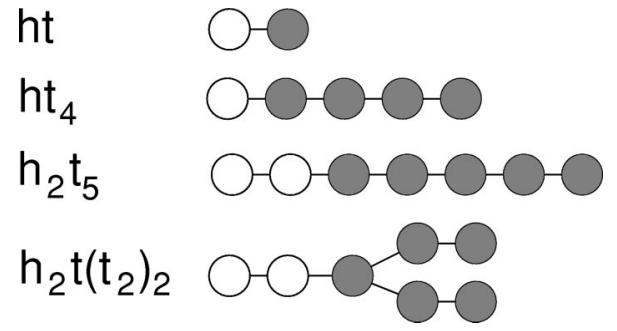

FIG. 1. Some of the model surfactants investigated in this study and the nomenclature used. The white particles are hydrophilic beads (h) and the gray particles hydrophobic tails $(\mathrm{t})$.

$$
\mathbf{F}_{i j}=\mathbf{F}_{i j}^{C}\left(r_{i j}\right)+\mathbf{F}_{i j}^{R}\left(r_{i j}\right)+\mathbf{F}_{i j}^{D}\left(r_{i j}\right),
$$

where the forces are of the form

$$
\begin{aligned}
& \mathbf{F}_{i j}^{C}=a_{i j} w^{C}\left(r_{i j}\right) \hat{\mathbf{r}}_{i j}, \\
& \mathbf{F}_{i j}^{R}=\sigma w^{R}\left(r_{i j}\right) \theta_{i j} \hat{\mathbf{r}}_{i j}, \\
& \mathbf{F}_{i j}^{D}=-\eta w^{D}\left(r_{i j}\right)\left(\hat{\mathbf{r}}_{i j} \cdot \mathbf{v}_{i j}\right) \hat{\mathbf{r}}_{i j} .
\end{aligned}
$$

Here, $\mathbf{v}_{i j}$ is the velocity difference for the two particles, $\hat{\mathbf{r}}_{i j}$ is the unit vector pointing from particle $i$ to particle $j . \theta$ is a random number between 0 and $1, a_{i j}, \sigma$ and $\eta$ determine the amplitude of the conservative, random and dissipative forces, respectively, while the $w^{\prime}$ 's are weight functions. To obey the fluctuation-dissipation theorem, we must have $w^{D}=\left(w^{R}\right)^{2}$, and the system temperature will follow from the relation between $\sigma$ and $\eta: \sigma^{2} / \eta=2 k T .{ }^{22}$ We use the same integration algorithm, weight functions, and parameters as Groot and Warren, ${ }^{23}$

$$
w^{C}(r)=w^{R}(r)=\sqrt{w^{D}(r)}=w(r),
$$

where

$$
w(r)=\left\{\begin{array}{l}
1-\frac{r}{r_{c}} \text { for } r<r_{c} \\
0 \quad \text { for } r \geqslant r_{c} .
\end{array}\right.
$$

Throughout this paper we use reduced units. $r_{c}$ is the unit of length, $k T$ (the temperature of the thermostat) is the unit of energy, and the mass unit is the mass of a DPD bead. In these units, $\sigma=3.0$ and $\eta=4.5$.

\section{B. Model}

We use a coarse-grained approach where one DPDparticle represents a group of atoms, or a liquid volume. Water beads, oil beads, head groups, and tail groups are denoted by w, o, h, and t, respectively. The tail beads are identical to the oil beads. Some of the model surfactants investigated are shown in Fig. 1. A surfactant molecule consists of head groups and tail groups connected by harmonic springs:

$$
\mathbf{F}_{i j}^{\text {Bond }}=-k_{s}\left(r_{i j}-r_{0}\right) \hat{\mathbf{r}}_{i j} .
$$

We choose $k_{s}=100$ and $r_{0}=0.7$. Water and oil are represented by a single bead for simplicity. One tail bead typically represents a few $\mathrm{CH}_{2}$ groups. ${ }^{24-26}$ The repulsion parameters used are shown in Table I. These are taken from Groot, ${ }^{25}$ 
TABLE I. Repulsion parameters $a_{i j}$. w= water bead, $\mathrm{o}=$ oil or tail group, and $\mathrm{h}=$ head group.

\begin{tabular}{cccc}
\hline \hline & w & o & h \\
\hline w & 25 & 80 & 15 \\
o & 80 & 25 & 80 \\
h & 15 & 80 & 35 \\
\hline \hline
\end{tabular}

except that we used $a_{\mathrm{oo}}=25$ instead of 15 . This change has been made to obtain similar bulk densities in the oil and in the water phase (see also Ref. 5).

\section{Simulation details}

The systems contained typically 32000 beads. The number of surfactant molecules used varied from 600 for the largest and up to 1400 for the smallest, of which not all are at the interface. This gave equilibrium areas of typically $22 \times 22$. Periodic boundary conditions were applied in all three directions. All simulations were performed at a bead density of 3.0 and with a time step of 0.03 .

After an equilibrium area was reached, each system was simulated for at least 8000 Monte Carlo steps. Each Monte Carlo step consists of either a series of DPD steps (between 1 and 200 with equal probability) or an attempt to change the box shape.

Figure 2 shows a snapshot of two monolayers with a total of $618 \mathrm{~h}_{2} \mathrm{t}_{5}$ surfactant molecules. The 16000 oil beads and 16000 water beads are not shown for clarity. Due to the high repulsion between hydrophilic and hydrophobic beads (Table I) all the surfactant molecules are at the interfaces. If the interfacial tension is very low, the film is not strictly flat and undulatory waves can be observed.
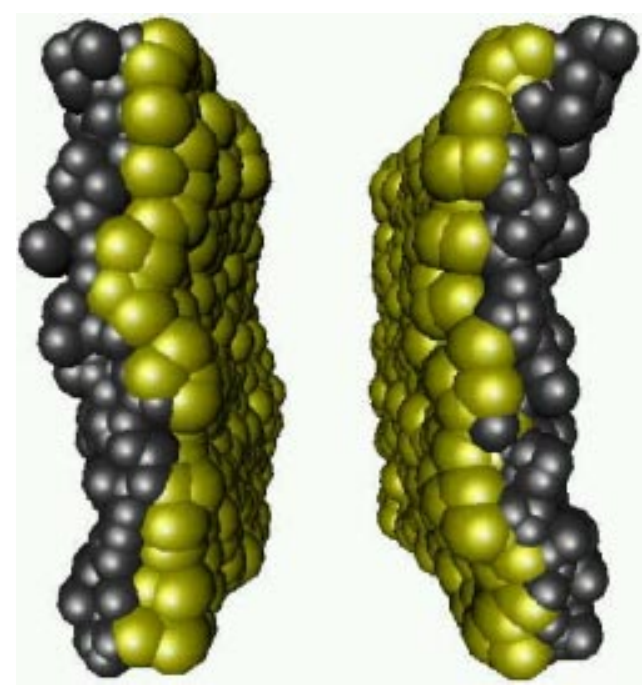

FIG. 2. Snapshot of $618 \mathrm{~h}_{2} \mathrm{t}_{5}$ surfactant molecules at zero interfacial tension. Head beads are in yellow and tail beads in gray. The head beads have been drawn larger compared to the tail beads to visualize the high head-head repulsion. Water (in the middle) and oil (on the sides) have been omitted for clarity. [Picture prepared with VMD (Ref. 27).] (Color online only.)

\section{Constant $N \gamma T$ conditions}

We perform most simulations in a constant $N \gamma T$ ensemble. The monolayers in microemulsions usually have ultralow interfacial tensions, but the area per surfactant molecule may vary depending on the surfactant. A constant $\gamma$ is thus a better reference state than constant area per molecule when comparing with experiments. Also, it is hard to impose a given area per molecule because not all surfactant molecules are necessarily at the interface; some may form micelles or dissolve in water.

By simulating a constant $N \gamma T$ ensemble we allow for the natural local area fluctuations in real systems. Lindahl and Edholm found that area fluctuations and undulations are weakly anticorrelated. ${ }^{10}$ This indicates that area fluctuations may influence the undulation intensity. We will therefore compare the constant area and constant interfacial tension schemes in Sec. II G.

Constant interfacial tension is achieved in the simulations by combining DPD with a Monte Carlo scheme. ${ }^{28}$ The box volume is kept constant while the shape of the box is allowed to fluctuate according to the specified $\gamma^{21}$

For surfactants on an oil/water interface, the interfacial tension depends on the amount and the type of surfactant. We find that $\gamma=0$ can be obtained with surfactants as small as $\mathrm{ht}_{3}$. Starting the simulation with a large area, the area will decrease until the surfactant density at the interface corresponds to that of zero interfacial tension. The tensionless interface will coexist with surfactant molecules dissolved in bulk or micelles. For ht and $\mathrm{ht}_{2}$ surfactants $\gamma=0$ could not be obtained. When imposing a too low interfacial tension in the simulations, an equilibrium box area cannot be established. The area continues to decrease while the surfactant molecules migrate into the water phase and eventually form various aggregates.

\section{E. Undulatory fluctuations}

To compute the bending moduli we analyze the fluctuations of the interface. The first step is to characterize the interface. For a sufficiently large number of surfactant molecules the interface can be described by continuum theory. ${ }^{4}$ Let $h(x, y)$ be the local displacement from the average position of the interface, $h(x, y)=z(x, y)-z_{0}$, where $z$ is the direction normal to the interface and $z_{0}$ is the average position of the interface. We will now rewrite Eq. (1) in terms of $h(x, y)$ as $h(x, y)$ can be monitored easily in the simulations. For small curvatures, $c_{1}+c_{2}=\nabla^{2} h$ and $d A$ $=d x d y \sqrt{1+(\nabla h)^{2}}$. For now we assume $c_{0}=0$ in Eq. (1). The surface integral over $c_{1} c_{2}$ is constant when the topology does not change, specifically, it is zero for a film. ${ }^{29}$ This implies that $\bar{\kappa}$ does not affect the energy fluctuations in a monolayer and henceforth not the calculations of the bending moduli. It also means that this elastic constant can not be determined by studying a given topology as in this study. The leading terms in curvature become ${ }^{30}$

$$
\begin{aligned}
e(h(x, y)) & \equiv(E(h(x, y))-E(0)) / A \\
& =\frac{\gamma}{2}(\nabla h(x, y))^{2}+\frac{\kappa}{2}\left(\nabla^{2} h(x, y)\right)^{2} .
\end{aligned}
$$


While the last term is the energy cost related directly to the bending of the monolayer, the first term on the right describes the energy cost due to an increase in area upon bending. Fourier transformation of Eq. (10) gives

$$
\widetilde{e}(\widetilde{h}(q))=\frac{\gamma}{2} q^{2} \widetilde{h}(q)^{2}+\frac{\kappa}{2} q^{4} \widetilde{h}(q)^{2} .
$$

According to the equipartition principle,

$$
\langle\widetilde{e}(\widetilde{h}(q))\rangle=\frac{1}{A} \frac{k_{B} T}{2}
$$

such that

$$
\left\langle|\widetilde{h}(q)|^{2}\right\rangle=\frac{k_{B} T}{A}\left(\gamma q^{2}+\kappa q^{4}\right)^{-1} .
$$

For short wavelengths the continuum picture is not valid. Molecular protrusions characterize the interface and these will be described by a protrusion tension $\sigma$ similar in nature to the interfacial tension, ${ }^{10}$

$$
\left\langle|\widetilde{h}(q)|^{2}\right\rangle=\frac{k_{B} T}{A}\left(\sigma q^{2}\right)^{-1} .
$$

Because we have applied a coarse-grained surfactant model, we will not investigate the interface at this level of detail.

Equation (13) predicts the spectral intensities of each undulation mode as a function of wave vector $q$. From the simulation we can obtain $\left\langle|\widetilde{h}(q)|^{2}\right\rangle$ by monitoring $h(x, y)$ and by fitting the results to Eq. (13), the elastic constants can be estimated.

\section{F. Spectral analysis}

To perform spectral analysis we need to monitor the local position $z(x, y)$ of each of the two interfaces. Note that we treat the two monolayers separately. The periodic boundary conditions prohibit simulation of a single monolayer between oil and water. A grid with $30 \times 30$ points was assigned to each interface. In the systems with high concentration of surfactants we defined the position of the interface locally from the positions of the surfactant head groups connected to the first tail bead. Two practical problems arise: First, how to determine whether a surfactant molecule belongs to one of the two interfaces or not. If it is dissolved in the bulk phases or is part of a micelle it should be disregarded when the position of the interface is calculated. Second, for each grid point $(i, j)$, the position $z(i, j)$ must be defined as some (weighted) average of the $z$-coordinates of the surfactant head groups nearby.

To determine which surfactant molecules belong to the interface, we used a cluster algorithm with the following criteria: Given two cut-off parameters $R_{x y}$ and $R_{z}$, two molecules $a$ and $b$ belong to the same cluster if

(5) $\left(z_{a}^{h}-z_{a}^{t}\right) /\left|z_{a}^{h}-z_{a}^{t}\right|=\left(z_{b}^{h}-z_{b}^{t}\right) /\left|z_{b}^{h}-z_{b}^{t}\right|$,
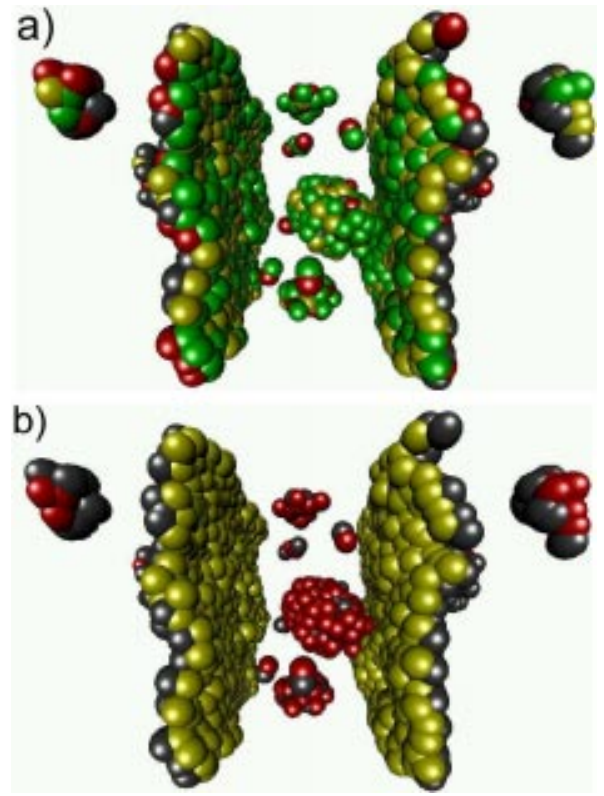

FIG. 3. Snapshots from a system with $50 \% \mathrm{ht}_{5}$ and $50 \%$ ht surfactant at interfacial tension $\gamma=0.1 \gamma_{0}$. Only the head groups and the first tail bead (the one connected to the head) are shown. The head beads have been drawn larger compared to the tail beads to visualize the high head-head repulsion. Water (in the middle) and oil (on the sides) as well as the four last tail beads of ht ${ }_{5}$ have been omitted for clarity. (a) and (b) represent the same configuration. In (a) beads are colored according to type: $\mathrm{ht}_{5}$ heads in yellow, $\mathrm{ht}_{5}$ tails in gray, ht heads in green, and ht tails in red. In (b) the head groups are colored yellow if they are assigned to the interface and red otherwise. [Picture prepared with VMD (Ref. 27).] (Color online only.)

where superscript $h$ denotes the head bead closest to the tail and $t$ the tail bead closest to the head. The four first criteria ensure that both the two head groups and the two tail groups are close. The last criterion states that their head-tail bonds must have the same direction projected onto the interfacial normal. This is efficient in filtering out single surfactant molecules or micelles very close to the interface. Different values for the parameters $R_{z}$ and $R_{x y}$ were used depending on the type of surfactant(s). This involved some trial and error until the two largest clusters correctly included all and only those surfactant molecules making up the interface. Visual inspection of snapshots in which molecules belonging to the two largest clusters were color-marked served as a final check. Typical values that gave a good description of the interface were $R_{z}=0.9-1.1$ and $R_{x y}=1.8-2.1$. For each surfactant type and density, we found a range in which small variations $\delta$ of $R_{z}$ and $R_{x y}$ did not affect the number of surfactant molecules in the interface. Then all the surfactant molecules at the interfaces are included in the cluster and those that are a distance between $R_{z}$ and $R_{z}+\delta$ away are filtered out with the bond direction criterion anyway.

Figure 3 shows an example configuration with $50 \% \mathrm{ht}_{5}$ and $50 \%$ ht surfactant at $\gamma=0.1 \gamma_{0}$ where $\gamma_{0}=3.45$ is the bare oil-water interfacial tension. Only the head groups and the tail groups attatched to them are shown for clarity. The small surfactant molecules are soluble in water and there are also a few micelles. In Fig. 3(a) the head groups of the large and small surfactant molecules are shown in yellow and green, respectively, the tails are in gray and red. In Fig. 3(b) 
the surfactants are colored according to the outcome of the cluster analysis: Surfactants that are determined to be part of the interface have yellow head groups, while the rest have red head groups.

The position $z(i, j)$ must be defined as some function of the $z$-coordinates of surfactant molecules close to the grid point, for example,

$$
z(i, j)=\frac{\sum_{k} r_{k}^{-p} z_{k}}{\sum_{k} r_{k}^{-p}}
$$

where $r_{k}=\left(x_{k}-\Delta i\right)^{2}+\left(y_{k}-\Delta j\right)^{2}$ and the sum is over all surfactant molecules that have $r_{k}<R$. $R$ must be large enough such that at least one molecule is assigned to the grid point but small enough such that $z(i, j)$ is indeed the local position. A high $p$ gives the molecules close to the grid point more weight. We chose $p=5$ and $R$ around unity to get $1-4$ surfactant molecules within $R$. We find that the values chosen for $R, p$ and the number of grid points affect the intensity of the highest $q$-modes (protrusion modes). However, they have only a negligible effect on the low $q$-modes from which we determine the bending modulus.

For the systems with few or zero surfactant molecules (the first four points in Fig. 5 below) the interface could not be described by the surfactants alone so we used a different definition of the interface. We let all pairs consisting of one hydrophobic and one hydrophilic bead which were closer than $r_{c}$ contribute to the interface. Now all surfactant molecules are at the interfaces so no cluster routine was needed. The assignment of positions to grid points were done in the same way as for high surfactant concentrations.

The two-dimensional discrete Fourier transformation yields

$$
\tilde{h}\left(i_{q}, j_{q}\right)=\sum_{i=0}^{n-1} \sum_{j=0}^{n-1} h\left(i_{r}, j_{r}\right) \exp \left\{\frac{-2 \pi i}{n}\left(i_{r} i_{q}+j_{r} j_{q}\right)\right\} .
$$

We can replace $h\left(i_{r}, j_{r}\right)$ by $z\left(i_{r}, j_{r}\right)$ in Eq. (16) as the constant $z_{0}$ vanishes in the summation. After averaging $\tilde{h}\left(i_{q}, j_{q}\right)$ over all snapshots we can obtain $\tilde{h}\left(q_{r}\right)$ for $q_{r}$ $=\sqrt{\left(i_{q}^{2}+j_{q}^{2}\right)} 2 \pi / L_{x}$, where $L_{x}$ is the average box size in $x$ and $y$ direction and $\left(i_{q}^{2}+j_{q}^{2}\right) \leqslant(n-1)^{2}$.

Figure 4 shows the spectral intensity $S(q)=\left\langle|\widetilde{h}(q)|^{2} A\right\rangle$ versus $q=2 \pi / \lambda$, where $A$ is the average box area and $\lambda$ is the undulation wave length for $\mathrm{h}_{2} \mathrm{t}_{5}$ surfactants at $\gamma=0$. The full line in Figs. 4(a) and 4(c) represents $S(q)=1 /\left(3.3 q^{4}\right)$. The agreement with Eq. (13) is very good: At long wavelengths, the continuum picture is valid and $S(q)=1 /\left(\kappa q^{4}\right)$. In the $\log / \log$ plot we can identify $q^{-4}$ behavior for low $q$ values and a transition towards $q^{-2}$ behavior for high $q$. When plotting $1 /\left(S(q) q^{2}\right)$ versus $q^{2}$ we get a straight line in the continuum regime, where $\kappa$ is the slope and $\gamma$ is the crossing with the $y$-axis.

The box dimensions limit the lowest $q$ mode we can measure to $q_{\text {min }}=2 \pi / \sqrt{A}$. We stress that Eq. (13) is valid in the continuum limit so $1 /\left(S(q) q^{2}\right)$ versus $q^{2}$ is only linear in the limit $q \rightarrow 0$. This means that there is some uncertainty in the extrapolation needed to determine $\kappa$. Thus a systematic error due to the finite system size might add to the statistical
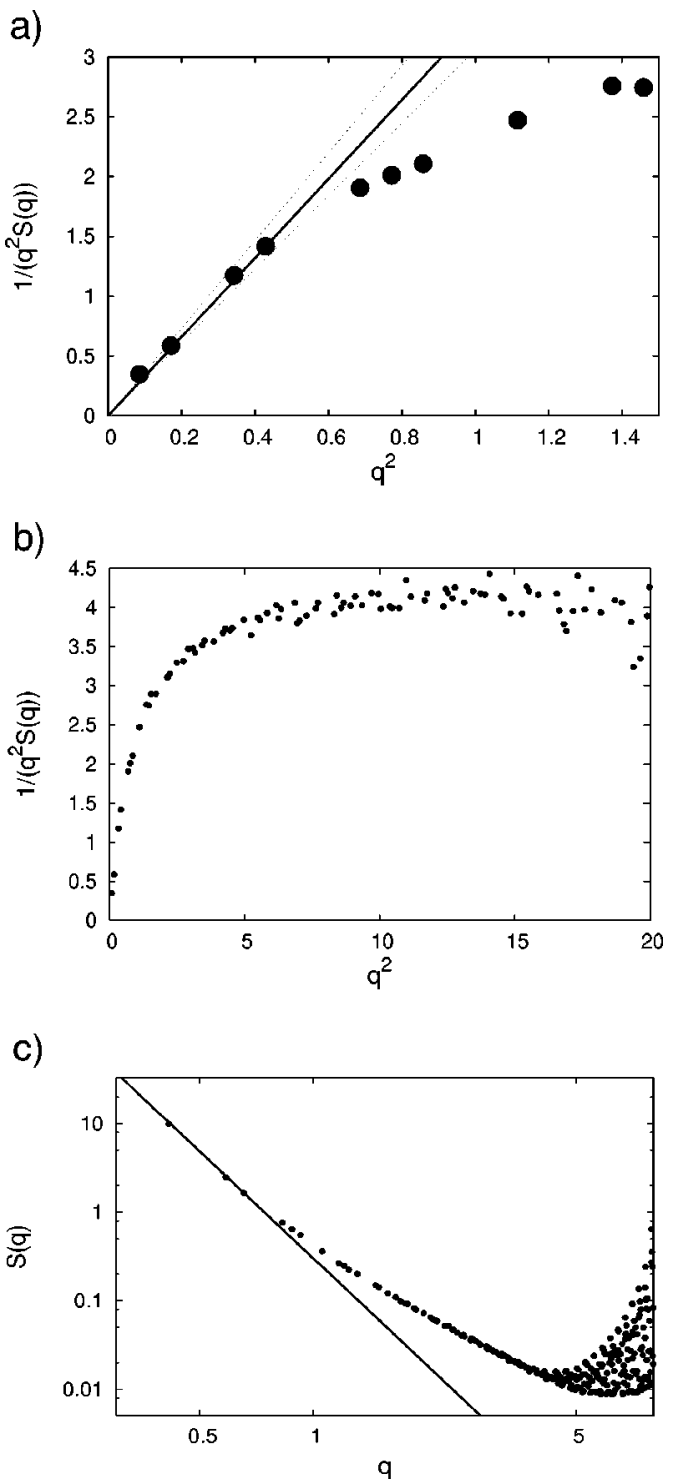

FIG. 4. Spectral intensities for $\mathrm{h}_{2} \mathrm{t}_{5}$ at $\gamma=0$. The slopes of the full and dotted lines represent the values for $\kappa$ and the lower and upper error bars in Fig. 11, respectively.

errors shown in the plots. All $\kappa$ values reported here have been calculated by fitting the first four points (weighted by their statistical errors) to $y=\gamma+\kappa x$. The reported error bars include results and uncertainties if we fit to three or five points.

\section{G. Comparison between constant $\gamma$ and constant A simulations}

To compare the results and accuracy in the constant interfacial tension and the constant area ensemble, we studied the dependence of $\kappa$ on interfacial density for the $\mathrm{ht}_{4}$ surfactant. First we performed constant area simulations with various surfactant concentrations. We sampled the interfacial tension and continued the simulations imposing this interfacial tension. We found no difference in results between the two ensembles within the uncertainty of the results (see Fig. 5 in Sec. III A). However, the accuracy differs. Because the constant interfacial tension is more time consuming, the $N V T$ ensemble is more efficient for a given CPU time, but 
for a given number of DPD steps, the $N \gamma T$ ensemble gives better accuracy. This is mainly because there is no uncertainty in $\gamma$, and the accuracy of $\gamma$ influences the accuracy in $\kappa$ when fitted to Eq. (13).

We perform most simulations in the $N \gamma T$ ensemble to compare different structures and compositions at the same (zero or very low) interfacial tension, resembling saturated monolayers and microemulsion conditions.

\section{H. Asymmetric surfactants}

Equation (10) is valid for interfaces where the preferred curvature is zero, for example bilayers or monolayers of surfactants which are symmetric in the sense that they do not prefer to bend towards either the oil or the water phase. The surfactants studied here are, like most real surfactants, asymmetric. However, in a simulation with periodic boundary conditions the interfaces are constrained to be flat on average. This means that the average curvature, $\bar{c}=\left\langle c_{1}+c_{2}\right\rangle / 2$ $=0$, differs from the preferred curvature $c_{0}$ which is determined by the characteristic volume $v_{0}$, chain length $l_{s}$ and head group area $a_{0}$ of the surfactant. ${ }^{4}$

The replacement of $c_{0}$ with $\bar{c}$ in Eq. (1) may in general lead to a distribution of modes differing from Eq. (13). This would be observed in the fluctuation spectrum with $S(q)$ differing from Eq. (13). Since the model fits the spectrum well, we neglect this error. Specifically, we can check the $q^{2}$ dependence since the interfacial tension is imposed and can also be calculated independently from the pressure tensor. ${ }^{31}$ We obtain the interfacial tension on the y-axis within the errors by extrapolating the linear part to $q=0$ [Fig. 4(a)]. We conclude that Eq. (13) is a good description of the fluctuation spectrum within the uncertainties of the method.

In real microemulsions where $\bar{c} \neq 0$ curvature fluctuations appear as deviations in droplet shape and size from spheres with radius $c_{0}^{-1}$. The size and shape fluctuations can be described in terms of spherical harmonics. ${ }^{32,33}$ That allows experimentalists to extract the bending moduli from analyzing the neutron scattering on microemulsion droplets. ${ }^{20,34}$ Those bending moduli describe the energy cost of curvature deviations away from the average droplet size with radius $c_{0}^{-1}$. The bending moduli reported here describe instead the average energy cost of curvature deviations away from a flat surface.

\section{RESULTS AND DISCUSSION}

We will here determine how the bending modulus depends on the molecular structure and composition of surfactants. First we investigate the dependence on density for a simple linear surfactant (Sec. III A). This is done using both the constant area and constant interfacial tension ensembles for comparison. The remaining simulations will be at a constant low interfacial tension to resemble the conditions in microemulsions. We use $\gamma=0$ or $\gamma=0.1 \gamma_{0}$ where $\gamma_{0}=3.45$ is the bare oil-water interfacial tension.

\section{A. Effect of surfactant density}

For the linear surfactant $\mathrm{ht}_{4}$ we studied the dependency on interfacial density. We performed both constant area

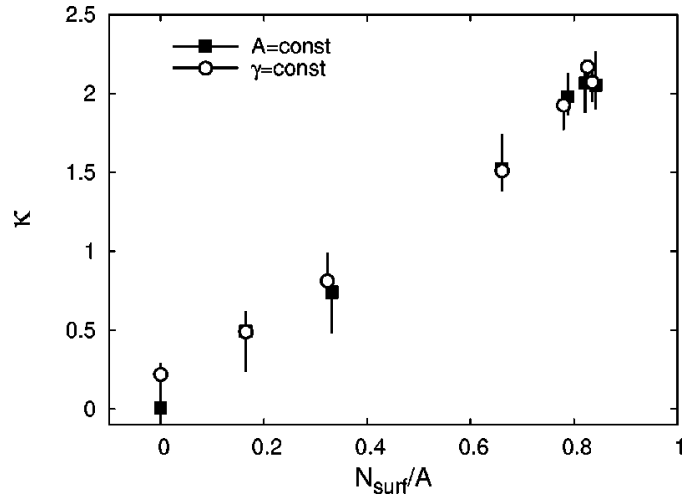

FIG. 5. Bending modulus as a function of surfactant density of $\mathrm{ht}_{4}$ surfactant. The simulations at constant interfacial tension (circles) are continuations of simulations at constant area (squares), imposing the average interfacial tension from the constant area simulations. Sampling was done during $5 \times 10^{5}$ DPD steps in both cases.

simulations and constant interfacial tension simulations. Figure 5 shows that the bending modulus increases monotonously with increasing density of surfactant. While the area fluctuations increase with increasing density due to lower interfacial tension, bending the sheet becomes increasingly energy costly. Without surfactant, bending of the layer has little effect on the packing, only on the area. The bending modulus is therefore very low. With surfactant at the interface, bending involves squeezing of the chains on one side of the layer. Oil and water molecules close to the surface can easily diffuse away from the compressed volume but the surfactant molecules cannot. As their density increases, steric forces due to bending increase accordingly.

A nonmonotonic dependence of $\kappa$ with area per surfactant molecule for weak linear surfactants was reported previously. ${ }^{14}$ Local fluctuations in the alignment at the interface can decrease the bending modulus from the bare oil/ water value. This effect disappears as the density increases and/or as the surfactant molecules become longer or stronger, i.e., when their tendency to align increases. For dense monolayers and/or strong surfactants the tendency to align perpendicular to the interface will increase monotonously with surfactant density. The surfactants investigated in this study, in particular the $\mathrm{ht}_{4}$ surfactant in Fig. 5, must be regarded as strong surfactants in the sense that the solubility in water ${ }^{5}$ is very low. Because of the coarse-graining they represent larger surfactants than the Lennard-Jones based four- and eight-beaded surfactants in Ref. 14. This might account for the qualitative difference observed.

\section{B. Effect of chain lengths}

We simulated monolayers of linear surfactants of type $\mathrm{ht}_{n}, n=1-6$ in the constant interfacial tension ensemble. A tensionless state could be obtained for $n \geqslant 3$. Figure 6 shows that the bending modulus increases linearly with the number of tail beads. As the monolayer becomes thicker, the energy cost of bending the monolayer increases. For monolayers of surfacants with a given area per molecule, one intuitively expects the energy cost of bending the membrane to increase as the tails become longer. Here we find that this is also true 


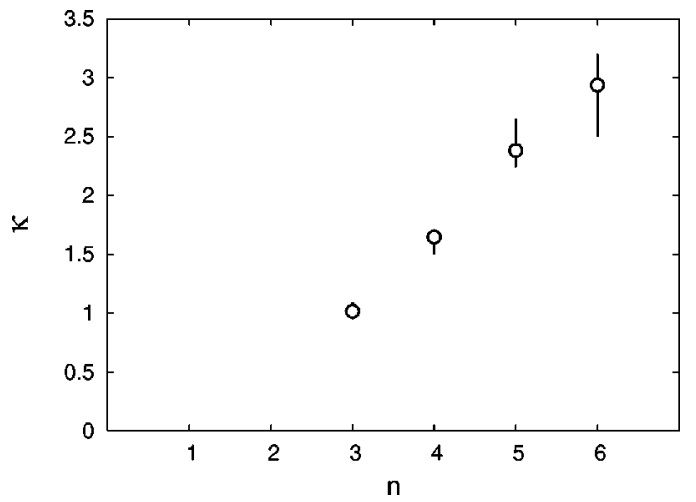

FIG. 6. Bending modulus as a function of the number of tail beads $n$ for $\mathrm{ht}_{n}$ surfactants at $\gamma=0$.

if one compares the different chain lengths at the same interfacial tension rather than interfacial density. This means that the chain length affects bending moduli more than the packing density does. Both $\gamma_{0}-\gamma$ and $\kappa$ increase with interfacial density and/or chain lengths. It is, however, interesting to note that $\kappa$ is more sensitive to these parameters than $\gamma$ is. For a given increase in chain length, the density can be lowered to give the same $\gamma . \kappa$, however, depends more strongly on chain length than density and will increase unless the density is further decreased.

Figure 6 suggests a linear increase with chain length in the given range. Theory predicts a power-law $\kappa \propto n^{p}, p$ $=2-3,4,16$ often used as a theoretical reference in experiments. ${ }^{34-37}$ However, in the theory the area per molecule is kept constant. That is not the case in our simulations nor in the experimental situation. The rate of increase in Fig. 6 would be higher if one compared different surfactants at a given density rather than at a given interfacial tension. This follows from two observations: The surfactant density at a given interfacial tension increases with increasing tail length, ${ }^{5}$ and the bending modulus increases with density for a given chain length (Fig. 5). Our results are in accordance with theory at similar conditions..$^{17,38}$

It is interesting to make a more detailed comparison with the experimental data. A tail bead in our model represents approximately three $\mathrm{CH}_{2}$ groups. The surfactants in Fig. 6 therefore correspond to chains with 9-18 alkyl units, which are typical lengths of real surfactants. $2 \kappa+\bar{\kappa}$ has been measured experimentally for surfactants with number of alkyl units between 8-12 (polyethyleneglycol alkyl ethers ${ }^{34,36}$ ) and $\quad 12-18 \quad(n$-alkyl- $n$-dodecyldimethylammonium bromides $^{37}$ and alkyl amine oxides ${ }^{36}$ ). The data are fit to power laws with $p=2.3$ and $p=2.95$ grounded on the theoretical prediction at constant area. However, with only 3-4 points for each surfactant type and some scattering in the results they could equally well fit a linear curve in the same range, especially because an investigation towards $n=0$ is impossible both in experiments and simulations (at $\gamma \simeq 0$ ).

\section{Effect of adding cosurfactant}

Figure 7 shows the bending modulus as a function of mole fraction of cosurfactant (ht) added to the $\mathrm{ht}_{5}$ surfactant. Also shown is the effect of reducing the chain length for all

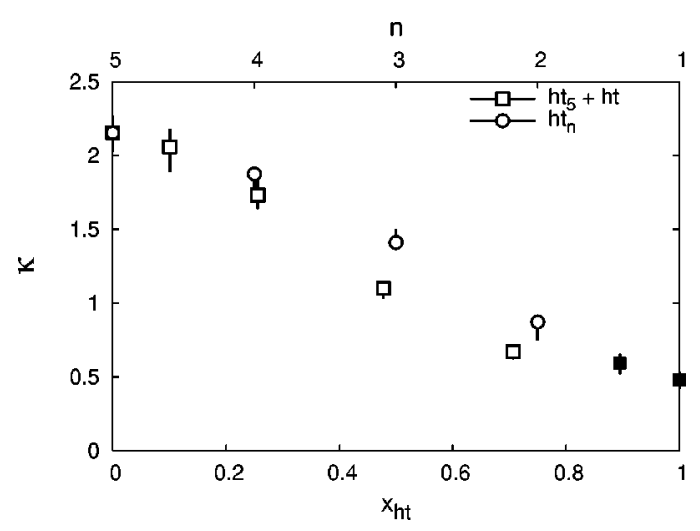

FIG. 7. Bending modulus as a function of mole fraction of cosurfactant added to the $\mathrm{ht}_{5}$ surfactants (squares, lower $x$-axis) and as a function of chain length $n$ for pure $\mathrm{ht}_{n}$ surfactants (circles, upper $x$-axis). The imposed interfacial tension was $\gamma=0.1 \gamma_{0}$ (open symbols) except for $x_{\mathrm{ht}}=0.9$ and $x_{\mathrm{ht}}=1.0$ which are at $\gamma=0.2 \gamma_{0}$ (solid symbols).

the surfactant molecules to the same average. We see that the effect of replacing some long surfactant molecules with short ones is larger than the effect of reducing the chain length of all molecules. A low tension monolayer cannot be obtained with all surfactant types, see discussion in Sec. II D. We therefore used $\gamma=0.1 \gamma_{0}$ except for $x_{\mathrm{ht}}=0.9$ and $x_{\mathrm{ht}}=1.0$ where we used $\gamma=0.2 \gamma_{0}$. Those two points are therefore slightly higher than the trend in Fig. 7 suggests.

The effect of binary mixture versus single-component monolayer was also investigated using mean-field theory assuming constant area ${ }^{15,16,19}$ and assuming saturated monolayers. ${ }^{17,18}$ Szleifer et al. ${ }^{15}$ predicted that the bending modulus of an equimolar mixture could become as low as that of a membrane composed of only the short molecules. Cantor ${ }^{17}$ found no pronounced difference between a mixed and pure monolayer at any average chain length. Our results are somewhat in between: A lower $\kappa$ for the binary mixture, decreasing with mole fraction of the ht surfactant. The calculations in Ref. 17 are carried out under the condition of "saturated" monolayers, which is similar to imposing a low interfacial tension. In Ref. 16 the monolayers are compared at a given area, i.e., not accounting for the variation in surface density. It is therefore interesting to note that our simulation results differ qualitatively from both these works.

The suppression of a possible spontaneous curvature $c_{0}$ might have an effect on the measured $\kappa$. Calculations have shown that the cosurfactant has a larger effect on the curvature than on the bending modulus. ${ }^{18}$ Here, a mean curvature of zero is forced through the periodic boundary conditions.

A reduction in bending rigidity with addition of short chain surfactant was also observed experimentally. Gradzielski et al. studied a binary mixture of $\mathrm{C}_{12} \mathrm{E}_{5}$ and $\mathrm{C}_{8} \mathrm{E}_{2}$ surfactant (polyethyleneglycol alkyl ethers) and found a trend and values very similar to those in Fig. $7 .{ }^{36}$ It was also found that the decrease in $\kappa$ upon adding cosurfactant depends on the difference in chain length between the short and long surfactants; short surfactants reducing $\kappa$ more for a given mole fraction. ${ }^{35,39}$

An interesting question is how $\kappa$ behaves at low mole fractions of ht. Figure 8 shows a linear decrease in the range 


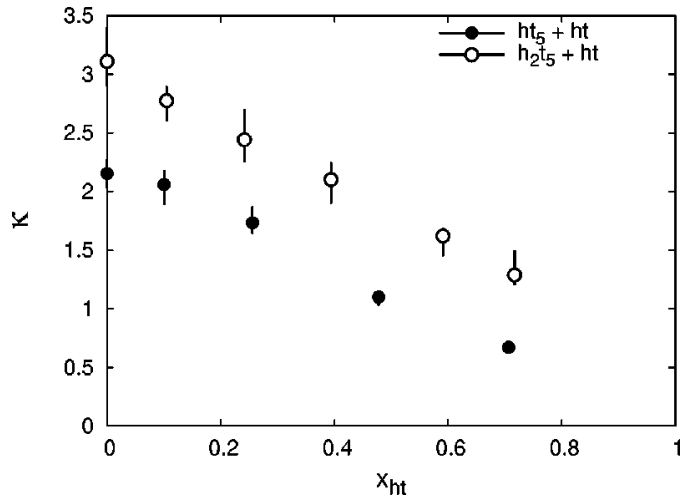

FIG. 8. Bending modulus as a function of mole fraction of cosurfactant added to $\mathrm{ht}_{5}$ and $\mathrm{h}_{2} \mathrm{t}_{5}$ surfactants at $\gamma=0.1 \gamma_{0}$.

of investigation. Mean-field theory at constant area predicts that the film can become very flexible by adding only a small amount of cosurfactant, i.e., a hyperbolic decrease in $\kappa$ with mole fraction. ${ }^{16,19}$ Without the constant area constraint the decrease is linear for $x<0.6 .{ }^{17}$ This is consistent with our results. The available experimental data also supports a linear decrease. ${ }^{36}$ It therefore seems that a constant area constraint overestimates the efficiency of cosurfactants in reducing the film rigidity.

We will now discuss the results of Fig. 7 in terms of the chain packing constraints. ${ }^{16,40}$ Short molecules acting as spacers between longer molecules reduce the chain-chain repulsion. That is particularly beneficial for a curved monolayer because the available area decreases towards the end of the chain, see Fig. 9. The cartoon shows how the available chain area changes with bending for surfactant molecules of uniform length and for a mixture between short and long surfactants. For those of uniform length, the area per chain segment increases close to the water phase and decreases towards the end of the chain compared to the flat layer. This is also the case for the mixed monolayer, but here this is highly beneficial as the density of chain segments is higher close to the water phase. This explains the difference between the two curves in Fig. 7. Figure 7 also suggests that the effect of the spacers is highest when the mole fractions are roughly equal. A snapshot from the simulation with $x_{\mathrm{ht}}$ $=0.5$ was shown in Fig. 3 .

To investigate possible ordering in the two-component monolayer we calculated the two-dimensional radial distribution function $g_{2 \mathrm{D}}(r)$ (Fig. 10). $r$ is here the distance be- a)

c)

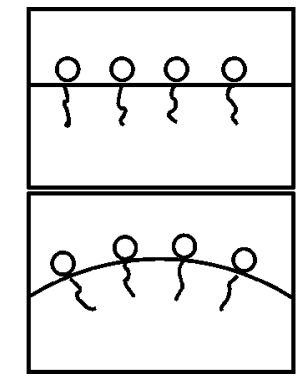

b)

d)

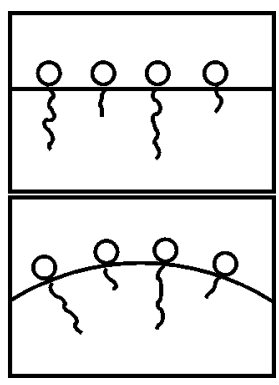

FIG. 9. Cartoon showing medium-length surfactants and a mixture of longand short-chain surfactants at a planar and curved interface.

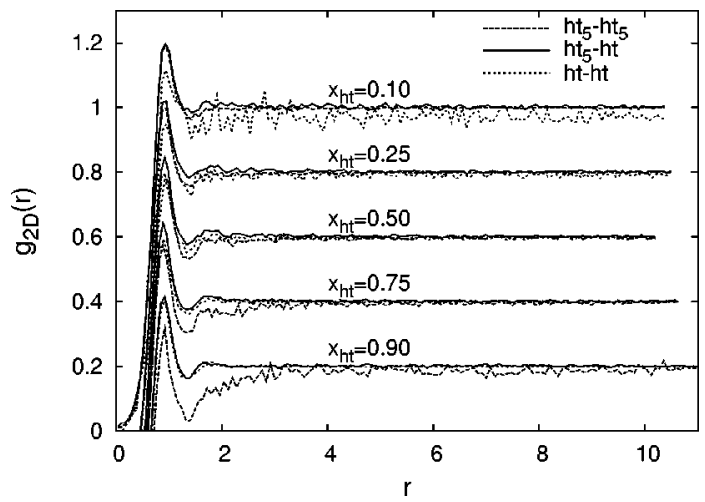

FIG. 10. Two-dimensional radial distribution function for head beads of pairs of $\mathrm{ht}_{5}-\mathrm{ht}_{5}, \mathrm{ht}_{5}-\mathrm{ht}$, and ht-ht molecules for various mole fractions of cosurfactant. Numbers on the $y$-axis refer to graphs for $x_{\mathrm{ht}}=0.10$. Graphs for the other mole fractions have been shifted down by $0.2,0.4,0.6$, and 0.8 , respectively.

tween two surfactant head groups projected onto the interface. Note that the absence of hard-core repulsion between DPD beads allow beads to overlap. Also, there is short-range order but no long-range order. The $\mathrm{ht}_{5}-\mathrm{ht}_{5}, \mathrm{ht}_{5}-\mathrm{ht}$, and ht-ht distributions differ for all mole fractions. This indicates short-range order in the distribution of the two types. The first peak is higher for pairs of different surfactants and the difference increases with decreasing difference in the mole fractions. This indicates a preference for $\mathrm{ht}_{5}$ molecules to be surrounded by ht molecules and vice versa. This is expected as the entropic repulsion between chains is higher for the surfactants with longer chains, and it supports the packing order shown in Figs. 9(b) and 9(d).

\section{Linear versus branched surfactants}

The difference between linear and branched surfactants with the same number of chain segments is interesting both from a theoretical and practical view point. Previously we reported that these branched surfactants are more efficient than the linear ones in reducing the interfacial tension: ${ }^{5} \mathrm{At}$ the same area density of surfactants, the branched give a lower interfacial tension. We will now determine the effect of branching on the bending modulus.

We simulated monolayers of the linear surfactant $h_{2} t_{5}$ and the branched surfactant $\mathrm{h}_{2} \mathrm{t}\left(\mathrm{t}_{2}\right)_{2}$ at $\gamma=0$ and at $\gamma$ $=0.1 \gamma_{0}$. The results are shown in Fig. 11. The linear surfactant has a higher bending modulus than the branched surfactant. Because the linear one has higher $\gamma$ for a given density, its packing is denser at a given $\gamma$. Due to the double chain of $\mathrm{h}_{2} \mathrm{t}\left(\mathrm{t}_{2}\right)_{2}$, we might expect it to have a bending modulus similar to $h_{2} t_{2}$ or $h_{2} t_{3}$ at the same interfacial tension. Given the linear increase in $\kappa$ with chain length (Fig. 6), the difference between branched and linear surfactants is as expected.

Cosurfactant was added to both linear and branched surfactants. This decreases the difference between the two. It has less effect on the branched surfactant because its double tail already acts as a kind of spacer. 


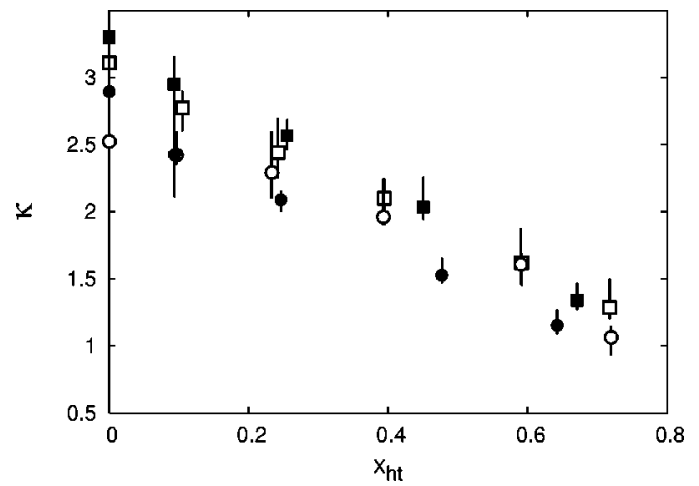

FIG. 11. Bending modulus for linear and branched surfactants as a function of mole fraction of ht surfactant added. The squares denote $h_{2} t_{5}+h t$ and the circles denote $\mathrm{h}_{2} \mathrm{t}\left(\mathrm{t}_{2}\right)_{2}+\mathrm{ht}$. The open symbols are at $\gamma=0.1 \gamma_{0}$ and the solid symbols are at $\gamma=0$.

\section{CONCLUSION}

One important result of this paper is that the thickness of the layer affects the rigidity more than the density of the layer: $\gamma$ decreases and $\kappa$ increases with both increasing density and increasing chain length. However, at given $\gamma$, i.e., accounted for a difference in density, $\kappa$ still increases with chain length.

Our simulations are performed at constant low interfacial tension, corresponding to microemulsion monolayers. We calculated values for $\kappa$ versus chain length for a onecomponent layer and versus mole fraction of cosurfactant for a binary mixture. The results differ qualitatively from theoretical predictions that assume constant area density. We find that $\kappa$ increases roughly linearly with chain length for a given head group. This is within the uncertainties of the experiments available, although these are often compared to theoretical predictions at constant area density. With this assumption the chain length increases faster. We also found that mixtures of short and long surfactants are more flexible than medium length surfactants of the same average chain length, but the decrease is roughly linear in mole fraction and less dramatic than without taking the density variations into account.

\section{ACKNOWLEDGMENTS}

These investigations are supported in part by the Norwegian Research Council (Grant No. 145184/432), by the Netherlands Research Council for Chemical Sciences (CW), and by the Netherlands Organization for Scientific Research (NWO) through PIONIER.
${ }^{1}$ N. Gov, A. G. Zilman, and S. A. Safran, Phys. Rev. Lett. 90, 228101 (2003).

${ }^{2}$ S. P. Moulik and P. K. Paul, Adv. Colloid Interface Sci. 78, 99 (1998).

${ }^{3}$ J. Sjöblom, R. Lindberg, and S. E. Friberg, Adv. Colloid Interface Sci. 65, 125 (1996).

${ }^{4}$ S. A. Safran, Statistical Thermodynamics of Surfaces, Interfaces, and Membranes (Addison-Wesley, Reading, 1994).

${ }^{5}$ L. Rekvig, M. Kranenburg, J. Vreede, B. Hafskjold, and B. Smit, Langmuir 19, 8195 (2003).

${ }^{6}$ J. N. Israelachvili and H. Wennerström, J. Phys. Chem. 96, 520 (1992).

${ }^{7}$ D. Langevin, Adv. Colloid Interface Sci. 34, 583 (1991).

${ }^{8}$ J. Daillant and M. Alba, Rep. Prog. Phys. 63, 1725 (2000).

${ }^{9}$ R. Goetz, G. Gompper, and R. Lipowsky, Phys. Rev. Lett. 82, 221 (1999).

${ }^{10}$ E. Lindahl and O. Edholm, Biophys. J. 79, 426 (2000).

${ }^{11}$ S. J. Marrink and A. E. Mark, J. Phys. Chem. B 105, 6122 (2001).

${ }^{12}$ G. Ayton and G. A. Voth, Biophys. J. 83, 3357 (2002).

${ }^{13}$ W. K. den Otter and W. J. Briels, J. Chem. Phys. 118, 4712 (2003).

${ }^{14}$ M. Laradji and O. G. Mouritsen, J. Chem. Phys. 112, 8621 (2000).

${ }^{15}$ I. Szleifer, D. Kramer, A. Ben-Shaul, D. Roux, and W. M. Gelbart, Phys. Rev. Lett. 60, 1966 (1988).

${ }^{16}$ I. Szleifer, D. Kramer, A. Ben-Shaul, W. M. Gelbart, and S. A. Safran, J. Chem. Phys. 92, 6800 (1990).

${ }^{17}$ R. Cantor, J. Chem. Phys. 99, 7124 (1993).

${ }^{18}$ R. Cantor, J. Chem. Phys. 103, 4765 (1995).

${ }^{19}$ S. May and A. Ben-Shaul, J. Chem. Phys. 103, 3839 (1995).

${ }^{20}$ M. Gradzielski, Curr. Opin. Colloid Interface Sci. 3, 478 (1998).

${ }^{21}$ M. Venturoli and B. Smit, Phys. Chem. Commun. 2, 45 (1999).

${ }^{22}$ P. Español and P. B. Warren, Europhys. Lett. 30, 191 (1995).

${ }^{23}$ R. D. Groot and P. B. Warren, J. Chem. Phys. 107, 4423 (1997).

${ }^{24}$ P. Prinsen, P. B. Warren, and M. A. J. Michels, Phys. Rev. Lett. 89, 148302 (2002).

${ }^{25}$ R. D. Groot, Langmuir 16, 7493 (2000).

${ }^{26}$ R. D. Groot and K. Rabone, Biophys. J. 81, 725 (2001).

${ }^{27}$ W. Humphrey, A. Dalke, and K. Schulten, J. Mol. Graphics 14, 33 (1996).

${ }^{28}$ S. M. Willemsen, T. J. H. Vlugt, H. C. J. Hoefsloot, and B. Smit, J. Comput. Phys. 147, 507 (1998).

${ }^{29}$ G. Gompper, Soft Matter, Complex Materials on Mesoscopic Scales, Vol. 10 in Matter and Materials, edited by J. K. G. Dhont, G. Gompper, and D. Richter (Forschungszentrum Jülich $\mathrm{GmbH}$, Institut für Festkörperforschung, Jülich, Germany, 2002), pp. B9.1-B9.46.

${ }^{30}$ W. Helfrich, Z. Naturforsch. A 33A, 305 (1978).

${ }^{31}$ D. Frenkel and B. Smit, Understanding Molecular Simulations: From Algorithms to Applications, 2nd ed. (Academic, San Diego, 2002).

${ }^{32}$ S. A. Safran, J. Chem. Phys. 78, 2073 (1983).

${ }^{33}$ S. T. Milner and S. A. Safran, Phys. Rev. A 36, 4371 (1987).

${ }^{34}$ M. Gradzielski, D. Langevin, and B. Farago, Phys. Rev. E 53, 3900 (1996).

${ }^{35}$ C. R. Safinya, E. B. Sirota, D. Roux, and G. S. Smith, Phys. Rev. Lett. 62, 1134 (1989).

${ }^{36}$ M. Gradzielski, D. Langevin, T. Sottmann, and R. Strey, J. Chem. Phys. 106, 8232 (1997).

${ }^{37}$ J. Eastoe, D. Sharpe, R. K. Heenan, and S. Egelhaaf, J. Phys. Chem. B 101, 944 (1997).

${ }^{38}$ C. Guerra, A. M. Somoza, and M. M. Telo da Gama, J. Chem. Phys. 109, 1152 (1998).

${ }^{39}$ M. Gradzielski, Langmuir 14, 6037 (1998).

${ }^{40}$ I. Szleifer, A. Ben-Shaul, and W. M. Gelbart, J. Phys. Chem. 94, 5081 (1990). 